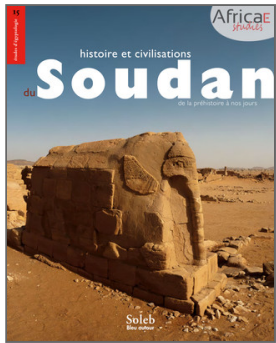

Histoire et civilisation du Soudan

De la préhistoire à nos jours

\title{
Carnets soudanais
}

\section{Olivier Rolin}

DOI : 10.4000/books.africae. 2757

Éditeur : Africae, Soleb, Bleu autour

Lieu d'édition : Paris, Khartoum

Année d'édition : 2017

Date de mise en ligne : 17 janvier 2022

Collection : Africae Studies

EAN électronique : 9782493207074

\section{(2) OpenEdition \\ Books}

http://books.openedition.org

Référence électronique

ROLIN, Olivier. Carnets soudanais In : Histoire et civilisation du Soudan : De la préhistoire à nos jours [en ligne]. Paris, Khartoum : Africae, 2017 (généré le 28 janvier 2022). Disponible sur Internet : <http:// books.openedition.org/africae/2757>. ISBN : 9782493207074. DOI : https://doi.org/10.4000/ books.africae. 2757 . 


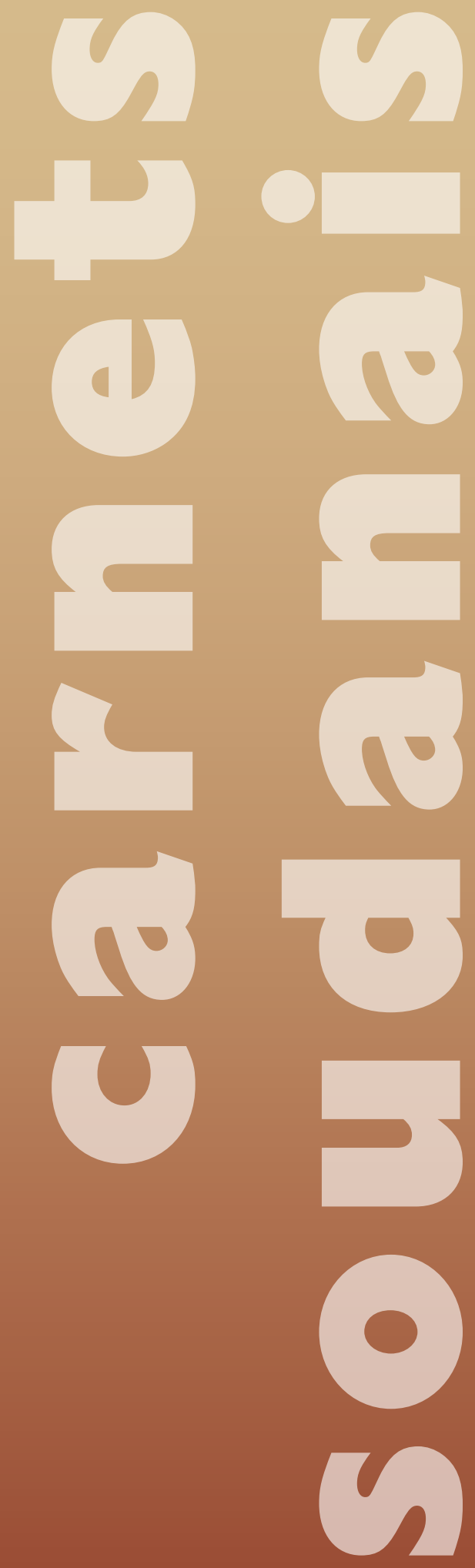




\section{J'ai quelques souvenirs forts du Soudan. Je les ravive en feuilletant de}

vieux carnets de notes écrits au crayon, à demi effacés. Sur les premières pages, j'avais recopié quelques versets particulièrement bien sentis du Coran, que j'avais lu à bord du Djoudi, un ferry saoudien faisant la ligne Suez-DjeddahSouakin («Vos femmes sont pour vous un champ de labour»; «Tuez les incrédules, partout où vous les trouverez»; «Tranchez la main du voleur et de la voleuse»; «Nous jetterons bientôt dans le feu ceux qui ne croient pas à nos signes, chaque fois que leur peau sera consumée nous leur en donnerons une autre», etc.). Ces citations, qui incidemment peuvent contribuer à éclairer certaines controverses actuelles, me furent d'un grand secours lors d'une interview que je fis pour Le Nouvel Observateur du cheikh Hassan al-Tourabi, un vieux filou corrompu qui faisait alors office de chef spirituel du Soudan, et me soutenait que la charia était l'avenir radieux du monde.

C'était pendant l'été 1994. J'avais écrit un roman dont j'avais situé l'action dans un Port-Soudan complètement imaginaire, et l'envie m’avait pris d'aller, juste avant qu'il ne paraisse, voir si la ville réelle ressemblait tant soit peu à la ville fictive, et plutôt infernale, que j'avais décrite. (Je m’apercevrai sans surprise que, si on ne saurait la recommander pour une lune de miel, par exemple, elle était tout de même nettement moins atroce que son double de papier.) Le Djoudi avançait vers un rivage bas. Au loin, on apercevait les hauteurs, aussi diaphanes que des nuages 
dans l'air brûlant, des Red Sea Hills. Debout sur un récif corallien, des hommes frappaient la mer avec de longs bâtons, comme Xerxès le fit sur l'Hellespont, mais probablement pour d'autres raisons. Les manœuvres d'accostage, sans l'aide d'un remorqueur, durèrent plus d'une heure. Des tortues nageaient dans les eaux vertes du bassin. Accoudé au bastingage en compagnie d'un marin indien mélancolique qui, m’ayant demandé mon nom, avait compris "Gulliver» en place d' "Oliver», j'eus tout loisir d'observer, reflétant ses minarets et ses maisons de corail dans l'eau lisse, hérissée, dentelée comme une ville bombardée, d'un blanc teinté de rose, l'antique Souakin sur sa presqu'île.

Les formalités d'immigration accomplies (mon passeport objet d'une curiosité multiplement minutieuse de la part de militaires lents et tatillons, et au demeurant fort polis, dans un hangar que le tournoiement de nombreux ventilateurs ne parvenait pas à rafraîchir), j'étais allé visiter les ruines de Souakin. Au-dessus de blocs de corail amoncelés, des porches ouvragés, des ogives brisées, des pans de mur portant encore des châssis de fenêtres, des souvenirs de persiennes, s'élançaient vers le ciel ardent. Des colonnes couchées miroitaient sous les friselis de l'eau. Des chèvres, des chats prenaient l'ombre sous des pans de murailles crénelées. Des milans perchaient sur le squelette d'une véranda. Des volées de petits oiseaux piaillards s'ébattaient autour du minbar d'une mosquée ouverte à tous vents. Un type 
charmant avait essayé de me vendre une mâchoire de requin qui semblait un piège à loups d'ivoire. Des silhouettes drapées d'indigo ou de pourpre glissaient silencieuses dans la blancheur incandescente, des barques effilées, sur l'émeraude de la mer. Mon premier contact avec le Soudan fut le spectacle, d'une beauté éclatante et funèbre, de ce Pompéi africain.

Ce ne fut pas le seul émerveillement, ni au cours de ce voyage ni au cours de deux autres que je fis ensuite. Car si on a compris sans doute que j'éprouve peu d'inclination pour le régime du Soudan et son idéologie islamiste, je dois dire que ce pays m’a prodigué des émotions qui me donnent parfois - en ce moment par exemple, tandis que je déchiffre mes vieux carnets - envie d'y retourner. Et ce ne sont pas seulement des lieux qu'il m'arrive d'avoir une nostalgie, mais des hommes aussi, certains en tout cas. Le chauffeur originaire des monts Nouba (qu'est-il devenu aujourd'hui, dans ce pandemonium sanglant qu'est l'histoire récente de la région?), qui conduisait à toute vitesse sa Land Cruiser à travers le désert, s'arrêtant pour faire ses ablutions et sa prière, mais estimant qu'aller à la mosquée, à l'église ou encore rester à la maison, c'était une affaire entre Dieu et soi dont le gouvernement n'avait pas à se mêler. Passant, à la sortie de Khartoum, devant une ancienne brasserie industrielle transformée en caserne, il me racontait le temps où, dans des guinguettes au bord du Nil, on mangeait de la friture en buvant de la bière: et Dieu, disait-il, n'y voyait rien de mal. (Comme on aimerait 
que ces vues soient plus universellement partagées!) Ou bien le vieux gardien à tête de chat ou de Chinois noir des ruines d'Old Dongola, à travers lesquelles volaient de grandes chauves-souris, cependant que nous devisions, assis sur des angarebs dans le soir qui tombait, salué par le roucoulement des tourterelles et le coassement des grenouilles. Ou bien encore cette femme soldat vêtue de vert olive, dont un léger voile noir cernait le beau visage, qui me fit par la grâce enjouée de son sourire trouver légère l'attente dans un des nombreux bureaux voués au contrôle des étrangers, et qui m’inspira un personnage de Méroé. Ou le vieil homme magnifique qui à Atbara me tendit une cruche d'eau pour laver mon visage encroûté de poussière rouge après sept heures passées dans la benne d'un «box", un de ces camions qui sillonnent le désert, avant d'insister pour payer mon passage dans l'autobus menant au «centre-ville» (si le mot convient). Comme avait aussi tenu à me faire voyager à l'œil, à Atbara toujours, un jeune chauffeur de taxi qui m’avait dit se sentir toujours heureux quand il voyait un étranger, et m’avait laissé son adresse afin que je lui envoie des catalogues de machines à presser l'huile d'occasion (je confesse que je ne l'ai pas fait, non par négligence mais par manque de relations dans les huileries).

Il serait exagéré de dire de Khartoum que c'est une des capitales les plus agréables du monde, mais ses larges rues poudreuses, tant bien que mal ombragées par le feuillage des neems, ont tout de même leur charme. 
Lorsque le haboob tend son voile de sable, tout, silhouettes enturbannées des hommes, drapés éclatants des femmes, ânes trottinant sous le bât et pick-up brinquebalant, y semble vu comme à travers la gaze sépia d'un écran de théâtre. Surtout, il y a les Nils, le blanc et le bleu, qui s'y rencontrent, l'un venu de la région des Grands Lacs, l'autre des hauts plateaux d'Éthiopie. Au confluent, il y avait à l'époque (et probablement toujours) un parc d'attractions d'une sympathique modestie. Des petites familles allongées sur l'herbe grillée, sous des Mickey et des Donald naïvement peints, des couples, des jeunes filles qui osaient saluer l'étranger d'un «hello!» joyeux (et peut-être moqueur) donnaient de Khartoum une image bonasse. Une grande roue qui n'avait de grande que le nom permettait tout de même de regarder de haut les carrés maraîchers de Tuti Island et la qubba argentée sous laquelle, à Omdurman, ne repose pas le Mahdi, puisque Kitchener, en 1898, fit exhumer et brûler son corps dans la chaudière d'une canonnière. Khartoum, plus que Le Caire, est à mes yeux la ville du Nil. C'est là qu'il naît à proprement parler. Il n'a pas encore été domestiqué, pompé, il est encore africain, il se souvient des forêts vierges et des grands marécages, des gorges et des montagnes de l'équateur. Le Nil Blanc en crue, c'est une mer de thé au lait, avec à l'horizon les pointillés des arbres, les griffures de quelques minarets. Plus étroit et rapide, le Nil Bleu file au ras de guinguettes où l'on boit du Coca-Cola (inutile de rêver à un gin tonic) en la jacasseuse compagnie d'ibis sacrés qui se rengorgent 
dans les arbres et vous conchient à l'occasion. En face, sur la rive de Khartoum North, les épaves des anciens steamers qui remontaient autrefois vers Juba et le cœur de l'Afrique encombrent ce qui reste du port fluvial. Lors de mon premier séjour, un fonctionnaire de la River Transport Corporation, que ma demande embarrassait (il se grattait la barbe, s'épongeait le front, évoquait l'autorité d'un autre chef dans un autre bureau, me renvoyait à tomorrow, se regrattait la barbe), avait fini, avec une amabilité lassée, par me laisser pénétrer sur ses quais. C'était le genre de panorama de décrépitude qu’aucun fonctionnaire, dans aucun pays, n'aime laisser visiter, un paysage qui invitait à la mélancolie.

À l'époque, il n'y avait pas de route asphaltée entre Shendi et Atbara (disant cela, j'ai l'impression de dater du temps du Soudan angloégyptien, et un doute me prend - mais comment expliquer, sinon, les sept heures passées pour faire les 135 kilomètres séparant les deux villes, et l'état dans lequel j'arrivai, transformé en statue de terre cuite et le cul pelé comme celui d'un babouin par les bonds du fût vide sur lequel j'étais assis dans la benne surpeuplée du box? Je me demande si la route qui existe aujourd'hui n'est pas due à la munificence de Ben Laden, qui fut l'hôte du pays de 1992 à 1996). Quoi qu'il en soit, c'est en se déhanchant sur des ornières sableuses qu’une Volga hors d'âge me mena pour la première fois à Méroé. Et ce fut un choc de découvrir, dessinant comme de formidables créneaux au sommet 
d'une dune, serrées l'une contre l'autre, les pyramides tronquées des cimetières royaux. Le vent sculptait entre elles des courbes douces, voluptueuses comme celles d'un corps, dans le sable orange semé d'éclats de pierre noire. On avait presque honte de fouler cette matière parfaite. Au loin vers l'ouest, du côté des temples et des palais de la cité royale, le soleil bas jetait des éclats de cuivre sur le Nil. Pas de route, pas de touristes: on pouvait presque se prendre pour Frédéric Cailliaud découvrant le site en 1821. J'ai fait, des années plus tard, d'autres visites à Méroé. Je me souviens d'y être arrivé, une fois, de nuit; les pyramides y dressaient comme de hautes voiles noires sous un ciel fourmillant d'étoiles. Je me souviens y avoir vu, assis au pied d'une pyramide du cimetière sud, le premier soleil passer au-dessus des dunes, époussetant le vert des maigres mimosées, teintant de mauve puis d'ocre le grès des chapelles funéraires, le vent du matin faire onduler comme une mousseline sur les courbes charnelles des coulées de sable: pour la plus grande joie des tourterelles qui s'envolaient en froufroutant et roucoulant, et la mienne, plus silencieuse.

Je ne suis nullement, on l'aura compris, un spécialiste du Soudan, pays sur lequel je n'ai écrit que des romans, et même, à strictement parler, un seul, puisque le pays de Port-Soudan est, je l'ai dit, un pays imaginaire. Mais «la liberté du romancier», écrivais-je en 1998 dans le post-scriptum de Méroé, «ne le délie pas d'un certain devoir d'étude 
et de rigueur ». J'ai donc fait autrefois ce que j'ai pu pour m'instruire de son histoire, aidé en cela par de longues lectures dans la bibliothèque du musée de Khartoum et la généreuse patience des savants que j'ai visités, Charles Bonnet à Kerma, Friedrich Hinkel à Méroé, Claude Rilly à Khartoum, notamment. Dans ce que j'ai appris de ses antiquités, ce qui m’a fasciné c'est l'anachronisme (je n'emploie pas ce mot dans le sens négatif qu'il a pris, mais dans celui, étymologique, qui désigne une marche à rebours du cours du temps). Aux yeux du profane, le Soudan ancien paraît une machine à remonter le temps, ce vieux rêve de l'Humanité. «C'est la grande fabrique des masques mortuaires, ici », faisais-je dire à un personnage d'archéologue dans Méroé. "Ou plutôt, beaucoup plus fort, l'atelier des momies vivantes. Regardez Méroé: ces gens, je veux dire ces rois, construisent des espèces de pyramides, adorent le dieu Amon et toute la clique, gravent des stèles dans une langue hiéroglyphique qu'ils ne comprennent plus, tout ça jusqu'au début du IV siècle. Cela fait presque un millénaire que l'Égypte pharaonique a cessé d'exister...» Et une chose comparable advient à l'époque médiévale avec les royaumes de Makouria et d'Alodia, tardifs rejetons de la Byzance de l'empereur Justinien et d'une Égypte chrétienne disparue depuis longtemps, que la conquête arabe a complètement coupés du monde où l'on adore la Croix. Rien de plus émouvant que de voir, sur les fresques conservées au musée de Khartoum, des archanges, des Vierges 
au visage de femmes minoennes, des évêques et des saints noirs peints dans un style qui évoque lointainement l'art russe des icônes, et dont la bouche laisse échapper des paroles en grec: « $\mathrm{Z} \Omega$ », «Je vis».

Beaucoup d'autres souvenirs encore se lèvent et se déploient à la lecture des pages presque effacées de mes carnets (quelque chose d'archéologique dans ce déchiffrement): la falaise sculptée de Gébel Barkal se dressant, mauve, dans le soir, espèce de rocher de Gibraltar sur la mer du désert; la stupeur devant la grande deffufa de Kerma, un monument tel que je n'en avais jamais vu, évoquant une cathédrale fondue; le labyrinthe incompréhensible (pour moi tout au moins) de Musawwarat; les lions de grès rouge de Naga, les ânes et les dromadaires, bien vivants ceux-là, qui tiraient d'un puits, près du temple d'Apédémak, des outres d'eau boueuse (et ce qui était beau, et qu'on voit difficilement ailleurs, dans d'autres pays, c'était la proximité des ruines anciennes et de la vie quotidienne, le grincement des poulies, le blatèrement des dromadaires entendus tout en contemplant les prisonniers de la callipyge candace Amanitoré levant vers elle une forêt de bras suppliants)...

Je referme ces carnets que je ne relirai peut-être jamais plus. J'ouvre ce livre où tout est décrit, avec l'exactitude et l'autorité que donne le savoir 